confinement. Further safety evaluation took place on Day 14. A Safety Review Committee reviewed safety, PK and PD data for each cohort of D-0120 dose level $(2.5 \mathrm{mg}, 5 \mathrm{mg}, 10 \mathrm{mg}, 20 \mathrm{mg}$ ) as well as when D-0120 $5 \mathrm{mg}$ was combined with $40 \mathrm{mg}$ febuxostat. PK evaluation for multiple dose parameters included AUC0-T, Cmax, Cmin, Tmax and FI.

Results: Dose escalation of D- 0120 from $2.5 \mathrm{mg} /$ day to $20 \mathrm{mg} /$ day was completed without any dose limiting toxicities. Most AEs occurred during the study were mild to moderate in severity and did not require any treatment before resolution. There was no SAE and no dose reduction during the treatment period. The pharmacokinetic (PK) evaluation of ascending dose levels of D-0120 suggested a dose proportional increase in drug exposure and there was no significant change of PK profile between Day 1 and Day 7 of dosing. For pharmacodynamic (PD) evaluation, the serum uric acid (UA) levels before and after D-0120 dosing was evaluated on multiple days. The UA reduction effect achieved maximum at about 4-8 hours after dosing and the effect lasted for at least 24 hours. After the 7-day dosing period, the mean percentage of UA reduction from baseline showed an increasing trend as the dose level increased.

More detailed safety, PK and PD data from multiple D-0120 dose cohorts and D-0120/febuxostat combination cohort will be presented at the meeting.

Conclusion: The oral daily administration of a novel URAT1 inhibitor, D-0120, in healthy volunteers for 7 days was well tolerated at dose levels from $2.5 \mathrm{mg} /$ day to $20 \mathrm{mg} /$ day. The PK profile demonstrated a dose proportional increase. D-0120 administration for 7 days resulted in significant reduction of serum UA levels. Further evaluation of this novel agent in longer treatment period and in patients with hyperuricemia and/or gout is warranted.

References: Not Applicable.

Disclosure of Interests: Ling Zhang Employee of: INVENTISBIO, David Wyatt: None declared, Kathryn Stazzone Employee of: INVENTISBIO, Zhe Shi Employee of: INVENTISBIO, Yaolin Wang Employee of: INVENTISBIO DOI: 10.1136/annrheumdis-2020-eular.5107

\section{OP0206 ALLANTOIN - A BIOMARKER OF OXIDATIVE STRESS - IS HIGHER IN PATIENTS WITH GOUT THAN IN HEALTHY VOLUNTEERS, AND CORRESPONDS WITH SEVERITY OF DISEASE}

L. Hasikova ${ }^{1,2}$, P. Kozlik ${ }^{3}$, K. Kalikova ${ }^{4}$, B. Stiburkova ${ }^{1,5}$, J. Zavada ${ }^{1,2} .{ }^{1}$ Institute of Rheumatology, Prague, Czech Republic; ' ${ }^{2}$ Department of Rheumatology, First Faculty of Medicine, Charles University, Prague, Czech Republic; ${ }^{3}$ Department of Analytical Chemistry, Faculty of Science, Charles University, Prague, Czech Republic; ${ }^{4}$ Department of Physical and Macromolecular Chemistry, Faculty of Science, Charles University, Prague, Czech Republic; ${ }^{5}$ Department of Pediatrics and Adolescent Medicine, First Faculty of Medicine, Charles University and General University Hospital in Prague, Prague, Czech Republic

Background: Uric acid can be non-enzymatically oxidized into allantoin and other products by reactive oxygen species. Allantoin has emerged as a reliable biomarker for monitoring oxidative status both in vitro and in vivo ${ }^{1}$. In gout patients, significantly increased plasma levels of allantoin have been found compared to healthy controls ${ }^{2}$.

Objectives: The aim of this pilot study was to measure allantoin as a biomarker of oxidative stress in patients with gout using newly developed UHPLCHILIC-MS/MS method ${ }^{3}$ and to investigate whether the allantoin levels are higher in patients with more severe disease (tophaceous gout).

Methods: We used clinical data and frozen serum $\left(-80^{\circ} \mathrm{C}\right)$ from 10 patients with chronic tophaceous gout, 10 patients with chronic gout without tophi and 10 healthy controls. Allantoin was determined in serum with sensitive UHPLC-MS/MS method using an isotopically labeled internal standard as we described before ${ }^{3}$. In addition, the concentrations of serum CRP, creatinine and uric acid were measured. Data are summarized as medians with interquartile range [IQR]. Differences between two patient groups were evaluated using the Wilcoxon signed-rank test.

Results: The median concentrations of allantoin in the serum from patients with tophaceous gout were significantly higher than in patients with gout without tophi (4.2 [2.6] vs. $3.2 \mu \mathrm{M}[1.5], p=0.0273$ ). There was no significant difference in other biochemical or demographic parameters (CRP, uric acid, creatinine, BMI, weight) between these two groups. Allantoin levels in healthy controls were significantly lower (0.5 vs. $4.2[2.6], p=0.0020,0.5$ vs. $3.2[1.5], p<0.0001$ ) (Fig. 1).

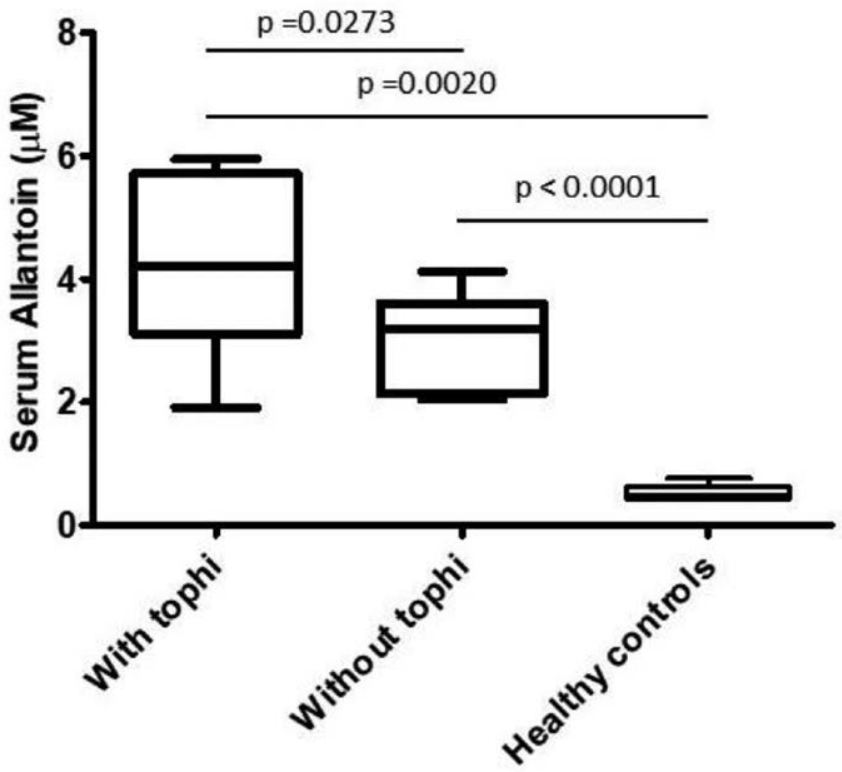

Fig. 1. Box plots of allantoin concentration in patients with tophaceous gout with tophi, without tophi and in healthy controls.

Conclusion: We have observed significantly higher levels of serum allantoin in the patients with more advanced gout with tophi compared with the patients with chronic gout without tophi. We have found elevated values of allantoin in both groups in comparison with healthy controls. In our small cohort the level of allantoin correspond with the severity of disease presented by tophi. However, further studies in large cohorts are needed. We can speculate, whether higher level of oxidative stress may contribute to increased cardiovascular risk and mortality in patients with gout (and more so in severe gout) ${ }^{4}$.

References:

[1] Marrocco I, Altieri F, Peluso I Measurement and Clinical Significance of Biomarkers of Oxidative Stress in Humans. Oxidative med and cell. longev. 2017:6501046.

[2] Stamp LK, Turner R, Khalilova IS, Zhang M, Drake J, Forbes LV, Kettle AJ. Myeloperoxidase and oxidation of uric acid in gout: implications for the clinical consequences of hyperuricaemia. Rheumatology (Oxford, England) 53 (11):1958-1965

[3] Kozlik P, Hasikova L, Stiburkova B, Zavada J, Kalikova K. Rapid and reliable HILIC-MS/MS method for monitoring allantoin as a biomarker of oxidative stress. Anal. Biochem. 2020 Jan 15; 589:113509

[4] Perez-Ruiz F, Martinez-Indart L, Carmona L, Herrero-Beites AM, Pijoan J Krishnan E. Tophaceous gout and high level of hyperuricaemia are both associated with increased risk of mortality in patients with gout. Ann Rheum Dis. 2014 Jan; 73(1):177-182.

Acknowledgments: This work was supported by the project (Ministry of Health, Czech Republic) for consensual development of research organization 00023728 (Institute of Rheumatology).

Disclosure of Interests: Lenka Hasikova: None declared, Petr Kozlik: None declared, Kveta Kalikova: None declared, Blanka Stiburkova: None declared Jakub Zavada Speakers bureau: Abbvie, UCB, Sanofi, Elli-Lilly, Novartis, Zentiva, Accord

DOI: 10.1136/annrheumdis-2020-eular.5517

\section{Cartilage - heal thyself!}

\section{OP0207 MECHANISM OF CHONDROPROTECTIVE EFFECTS OF 2-DEOXYGLUCOSE}

K. Terabe ${ }^{1}$, N. Takahashi ${ }^{1}$, O. Yoshifumi ${ }^{1}$, M. Masataka ${ }^{1}$, W. Knudson ${ }^{2}$ C. Knudson ${ }^{2}$, T. Kojima ${ }^{1}$, N. Ishiguro ${ }^{1}$. ${ }^{1}$ Nagoya University Graduate School of Medicine, Orthopedics, Nagoya, Japan; ${ }^{2}$ East Carolina University, Greenville, United States of America

Background: We recently reported that the inhibitor of hyaluronan (HA) biosynthesis, 4-methylumbelliferone (4-MU) blocked IL-1 $\beta$ activation of MMP13 mRNA and protein expression in human osteoarthritic (OA), bovine as well as bovine or OA cartilage explants [1]. This was a somewhat counterintuitive observation 